\title{
Recent results from the long-baseline (LBL) neutrino oscillation experiments
}

\author{
Pablo Fernández ${ }^{\star}$
}

IFIC (CSIC \& University of Valencia), Valencia, Spain

$\star$ pablo.fer@cern.ch

Proceedings for the 15th International Workshop on Tau Lepton Physics, Amsterdam, The Netherlands, 24-28 September 2018

doi:10.21468/SciPostPhysProc.1

\begin{abstract}
The results from the current long-baseline neutrino oscillation experiments are reviewed. These experiments are designed to measure the remaining unknown neutrino oscillation parameters via the oscillation of accelerator neutrinos over long distances.

The performance of these experiments and their results are described and discussed, giving a precise picture of the current status of the measurements for the neutrino mass ordering, the value of $\Delta m_{32}^{2}$, the $\theta_{23}$ and the lepton CP-violating phase, $\delta_{C P}$.
\end{abstract}

(c) (1) Copyright P. Fernández.

This work is licensed under the Creative Commons

Attribution 4.0 International License.

Published by the SciPost Foundation.
Received 31-10-2018

Accepted 07-01-2019

Published 20-02-2019

doi:10.21468/SciPostPhysProc.1.029

\section{Contents}

1 Introduction 1

2 Physics of Neutrino Oscillations $\quad 2$

3 LBL Neutrino Oscillation Experiments $\quad 4$

3.1 The T2K Experiment 5

3.2 The NO $v$ A Experiment 9

3.3 T2K and NO $v$ A Joint Neutrino Oscillation Analysis 13

$\begin{array}{lll}3.4 & \text { The MINOS/MINOS+ Experiment } & 13\end{array}$

4 Results from Current LBL Neutrino Oscillation Experiments 16

5 Conclusions 16

$\begin{array}{ll}\text { References } & 17\end{array}$ 


\section{Introduction}

Long-baseline (LBL) neutrino oscillation experiments have provided a great progress in our knowledge of the neutrino properties compared to the experiments measuring neutrinos from natural sources. Thanks to their efficient design and operation, they have been able to get us closer to the measurement of the most challenging neutrino oscillation parameters, those are the $\theta_{23}$ mixing angle, the neutrino mass ordering and the CP-violating phase of the lepton sector.

In this conference paper the current status of the LBL neutrino oscillation experiments. First, in section 2, reviewing and summarizing the physics of neutrino oscillations with its main remaining unknowns, and how LBL are built so they can provide the most precise measurements for these unknown parameters. Then in section 3, the current LBL experiments are reviewed and their results discussed to give an overall picture of the current status of neutrino oscillation physics. The T2K, NO $v$ A and MINOS/MINOS+ experiments are described in subsections 3.1, 3.2 and 3.4. The OPERA experiment was covered by a dedicated talk in this same conference, so it is not addressed here.

Finally, the results of all these experiments are summarized in section 4 and the most important measurements from these experiments are highlighted in section 5.

\section{Physics of Neutrino Oscillations}

The first evidence of neutrino oscillations was provided by the Super-Kamiokande experiment in 1998 and via the analysis of atmospheric neutrinos. This confirmed the theoretical hypothesis of neutrino oscillations done by B. Pontecorvo in 1957.

The neutrino oscillations arise from the fact that neutrinos are not massless as assumed by the Standard Model (SM), making neutrino oscillations the first evidence of physics beyond the SM. The SM predicts the existence of three neutrinos and this is the framework assumed for the rest of this paper.

Neutrinos do not share the same flavor (interaction) and mass (propagation) eigenstates and they are transformed by a non-diagonal unitary matrix with four degrees of freedom, parametrized by three mixing angles $\theta_{12}, \theta_{13}$ and $\theta_{23}$ and a complex CP-violating phase, $\delta_{C P}$. These flavor and mass eigenstates are mixed through the PMNS matrix,

$$
\begin{gathered}
\left(\begin{array}{l}
v_{e} \\
v_{\mu} \\
v_{\tau}
\end{array}\right)=U_{P M N S}\left(\begin{array}{l}
v_{1} \\
v_{2} \\
v_{3}
\end{array}\right), \\
U_{P M N S}=\left(\begin{array}{ccc}
1 & 0 & 0 \\
0 & c_{23} & s_{23} \\
0 & -s_{23} & c_{23}
\end{array}\right)\left(\begin{array}{ccc}
c_{13} & 0 & s_{13} e^{-i \delta_{C P}} \\
0 & 1 & 0 \\
-s_{13} e^{i \delta_{C P}} & 0 & c_{13}
\end{array}\right)\left(\begin{array}{ccc}
c_{12} & s_{12} & 0 \\
-s_{12} & c_{12} & 0 \\
0 & 0 & 1
\end{array}\right),
\end{gathered}
$$

where $c_{i j}=\cos \theta_{i j}$ and $s_{i j}=\sin \theta_{i j}$.

This means that after neutrinos have propagated freely through space they can be detected with a different flavor from that of their origin. This phenomenon is called neutrino oscillations and the probability of a neutrino to change its flavor after a free propagation in vacuum is given by the following expression:

$$
P_{v_{l} \rightarrow v_{l^{\prime}}}(L / e) \approx \sum_{i j} U_{P M N S}^{l^{\prime}, i}\left(U_{P M N S}^{l, i}\right)^{*}\left(U_{P M N S}^{l^{\prime}, j}\right)^{*} U_{P M N S}^{l, j} e^{-i \frac{\Delta m_{i j}^{2} \frac{L}{E}}{E}}
$$




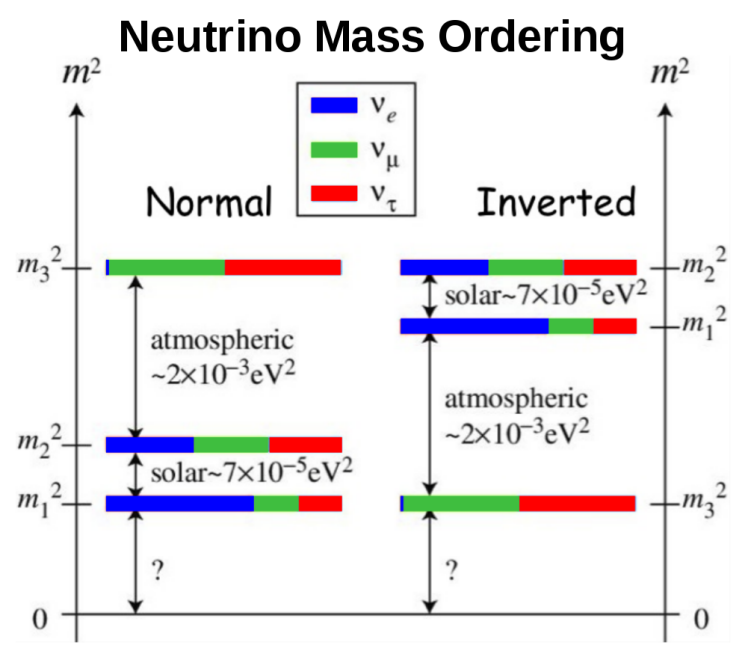

Figure 1: Two possible neutrino mass orderings allowed.

This means that the neutrino oscillation probabilities depend on the four parameters of the PMNS matrix, the ratio between the distance traveled by the neutrinos and their energy and, also, on two independent mass square differences.

In the three flavor neutrino framework, there are six independent oscillation parameters, four from the PMNS matrix and the two neutrino square mass differences.

$$
\Delta m_{21}^{2}=m_{2}^{2}-m_{1}^{2}, \Delta m_{31}^{2}=m_{3}^{2}-m_{1}^{2} .
$$

The third mass square difference is just a linear combination of the other two $\Delta m_{32}^{2}=\Delta m_{31}^{2}-\Delta m_{21}^{2}$.

This definition allows the existence of two possibilities for the ordering of the neutrino masses, normal (neutrino third mass eigenstate is the heaviest) and inverted (neutrino third mass eigenstate is the lightest), as shown in figure 1.

As LBL experiments mainly produce muon (anti)neutrinos to be propagated to the detector, there are basically two oscillation channels that matter for measuring the oscillation parameters.

The disappearance channel is the survival probability of the produced muon (anti)neutrinos at the accelerator facility. This channel is most sensitive to the $\theta_{23}$ and the neutrino mass ordering.

$$
P\left({\stackrel{(-)}{v_{\mu}}}_{\mu}{\stackrel{(-)}{v_{\mu}}}^{-} \cong 1-4 \sin ^{2} \theta_{23} \cos ^{2} \theta_{13}^{M}\left(1-\sin ^{2} \theta_{23}^{2} \cos ^{2} \theta_{13}^{M}\right) \sin ^{2}\left(\tilde{\Delta} \frac{L}{E}\right),\right.
$$

where

$$
\begin{array}{r}
\tilde{\Delta}=\frac{\Delta m_{32}^{2}+\Delta m_{21}^{2} \sin ^{2} \theta_{12}+\Delta m_{21}^{2} \cos \delta_{C P} \sin 2 \theta_{12} \sin \theta_{13} \tan \theta_{23}}{4}, \\
\sin ^{2} \theta_{13}^{M}=\frac{\sin ^{2} 2 \theta_{13}}{\sin ^{2} 2 \theta_{13}+\left(A-\cos 2 \theta_{13}\right)^{2}}, A=\sqrt{2} G_{F} N_{e} \frac{2 E}{\Delta m_{31}^{2}},
\end{array}
$$

and $N_{e}$ is the electron density of the Earth.

The appearance channel, on the other hand, is the probability of the muon (anti)neutrinos to be transformed into electron (anti)neutrinos. This channel is most sensitive to the $\theta_{13}$ mixing 
angle, which dominates the expression, the neutrino mass hierarchy and to the CP-violating phase, $\delta_{C P}$.

$$
\begin{aligned}
P\left(\left(_{\mu}^{(-)} \rightarrow\left(\stackrel{v}{v}_{e}\right) \cong\right.\right. & \underbrace{\sin ^{2} \theta_{23} \frac{\sin ^{2} 2 \theta_{13}}{(A-1)^{2}} \sin ^{2}\left((A-1) \Delta \frac{L}{E}\right)}_{\text {leading term, } \theta_{13}}+\alpha^{2} \cos ^{2} \theta_{23} \frac{\sin ^{2} 2 \theta_{12}}{A^{2}} \sin ^{2}\left(A \Delta \frac{L}{E}\right) \\
& +\frac{\alpha J_{C P}}{A(1-A)} \sin \left(A \Delta \frac{L}{E}\right) \sin \left(\Delta(1-A) \frac{L}{E}\right)(\underbrace{\cot \delta_{C P} \cos \left(\Delta \frac{L}{E}\right)}_{\text {CP conserving term }} \underbrace{\sin \left(\Delta \frac{L}{E}\right)}_{\text {CP violating term }}),
\end{aligned}
$$

where

$$
\alpha=\frac{\Delta m_{21}^{2}}{\Delta m_{31}^{2}}, \Delta=\frac{\Delta m_{31}^{2}}{4}, J_{C P}=\cos \theta_{13} \sin 2 \theta_{12} \sin 2 \theta_{23} \sin 2 \theta_{13} \sin \delta_{C P} .
$$

The expressions above differ from those obtained from equation 3 as they contain additional terms due to the fact that neutrinos do not travel through vacuum, but through the Earth's crust. In terms of oscillation probabilities, this only affects to the electron neutrinos and antineutrinos as they are the only ones able to interact via coherent forward scattering with the electrons present in matter, as seen in equation 6.

\section{LBL Neutrino Oscillation Experiments}

The basic setup of current LBL neutrino experiments consists of near and far detectors to which (anti)neutrinos produced at accelerators are thrown.

Since the neutrino probabilities are modulated by $L / E$ (baseline over neutrino energy) ratio, LBL experiments are designed so this ratio is such that the disappearance and appearance channels are optimized.

The goal of long-baseline neutrino experiments is to measure the remaining neutrino unknown parameters, with special focus on the CP phase due to its implications, being a potential explanation for the matter-antimatter imbalance in our universe.

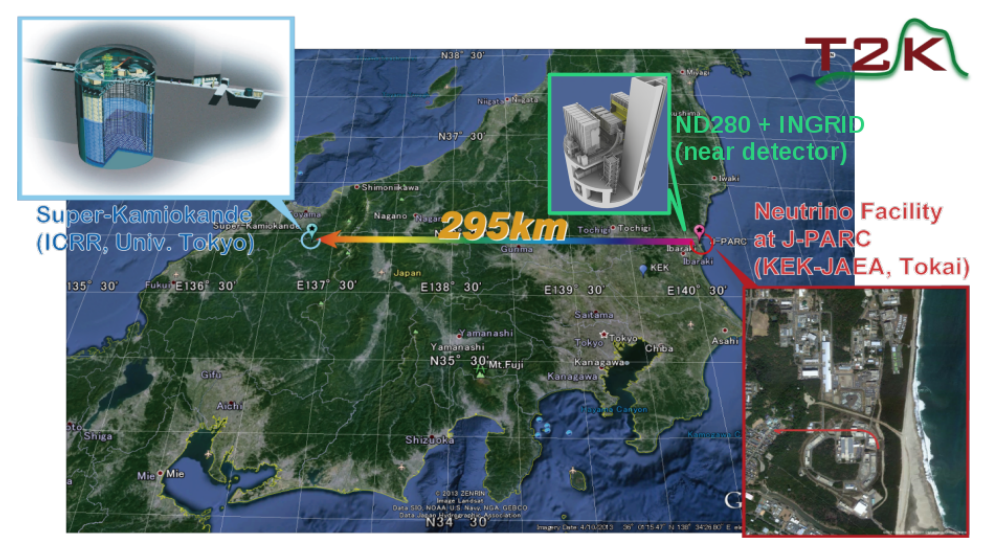

Figure 2: Geographical view of the T2K experiment layout. 


\subsection{The T2K Experiment}

The T2K experiment is located in Japan and its focus is in the measurement of the $\theta_{23}, \theta_{13}$ and $\delta_{C P}$. It uses the J-PARC neutrino facility at Tokai, which produces mainly $v_{\mu}$ and $\bar{v}_{\mu}$, depending on the beam mode. These neutrinos are then detected at the ND280 near detector facility and the Super-Kamiokande far detector.

\section{The T2K beam}

The J-PARC neutrino beam has been operating this year with a mean power of $485 \mathrm{~kW}$, achieving maxima of more than $500 \mathrm{~kW}$.

The total amount of protons-on-target (POT) accumulated during the T2K operation is $3.2 \cdot 10^{21}$, $1.51 \cdot 10^{21}$ in neutrino mode and $1.65 \cdot 10^{21}$ in antineutrino mode.

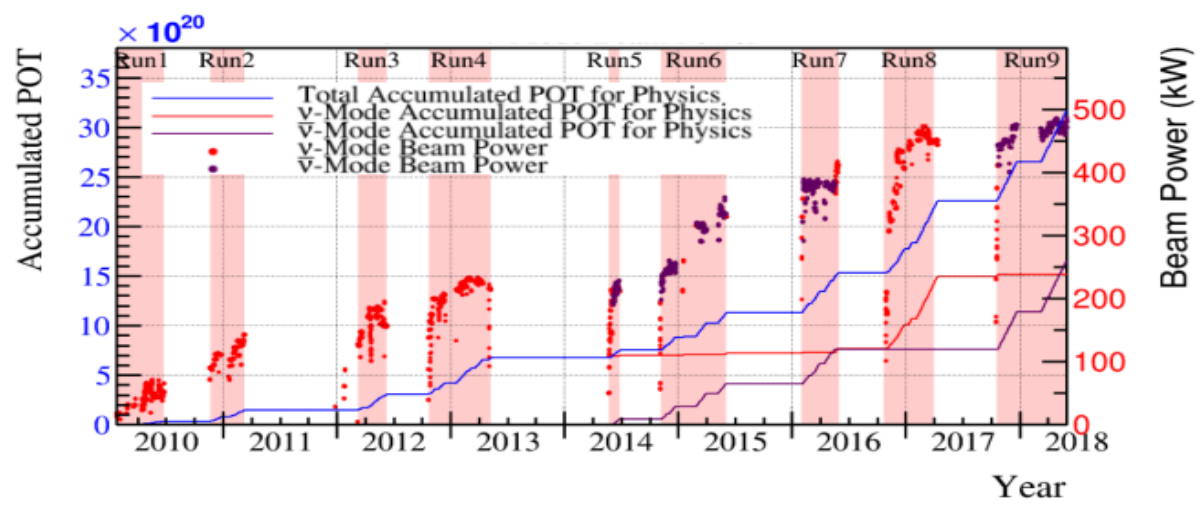

Figure 3: Accumulated POT and neutrino beam power output during the T2K operation.

The direction of the beam is not aligned with the ND280 near detector and the SuperKamiokande far detector, but $2.5^{0}$ off-axis. T2K was the first experiment to implement this technique due to its advantages. As shown in figure 4, with the $2.5^{0}$ off-axis setup, the neutrino spectrum is narrower than for other configurations peaking at $0.7 \mathrm{GeV}$, just where the maximum of the appearance channel and the minimum of the disappearance channels are.

Given to the enormous success during the T2K operation, it has been agreed to extend the operation of the J-PARC neutrino beam until 2026 with the aim of achieving $2 \cdot 10^{22}$ POT. This will suppose the extension of the T2K experiment, T2K phase-II.

\section{The T2K detectors}

T2K has a near detector as well, which performs cross-section, energy spectrum and flux studies of the neutrino beam. This provides great knowledge about the neutrino characteristics, decreasing the associated systematic errors. ND280 is a compendium of various facilities, placed $280 \mathrm{~m}$ from J-PARC. It is formed by INGRID and ND280. 


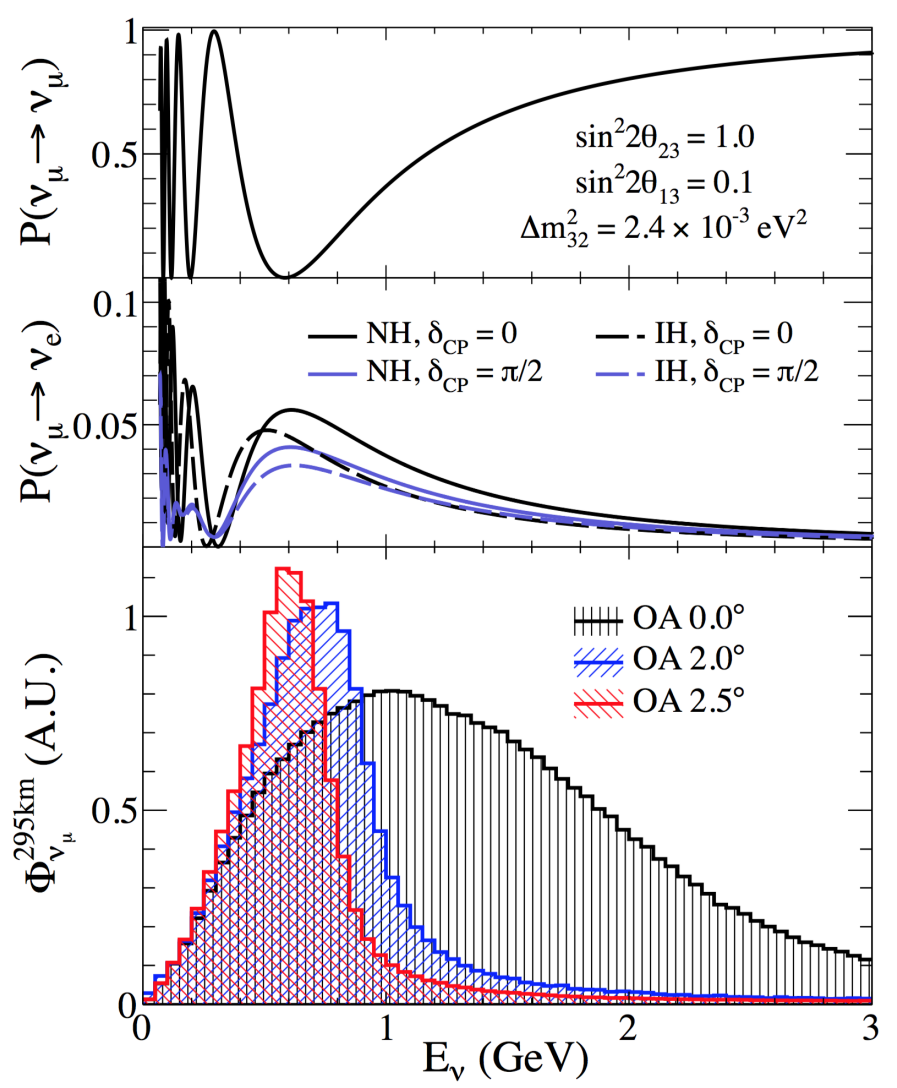

Figure 4: The plots show the T2K disappearance and appearance channel probabilities as well as the neutrino spectrum at the far detector assuming different off-axis configurations.

- ND280: This is placed $2.5^{0}$ off-axis and composed of $\pi^{0}$ detector and calorimeter, an electromagnetic calorimeter, a muon detector, fine grained detectors (FGD) and argon time projection chambers (TPCs). It will be upgraded for phase-II to reduce systematics up to $4 \%$.

- INGRID: This detector is on-axis and is a scintillation light detector made up by sixteen modules of iron plates and and eleven tracking scintillators each.

Due to the high neutrino flux at the near detector, its data is very useful to carry out studies concerning neutrino cross-section and studies to reduce the systematic errors related to the neutrino flux and interactions at the far detector.

Super-Kamiokande is the far detector, a water-Cherenkov detector with 50 kton of ultrapure water located in the Kamioka mine under $1000 \mathrm{~m}$ of rock (2700 m.w.e.). It is located $295 \mathrm{~km}$ from the neutrino beam and optically divided into inner and outer detector.

- The inner detector is used for physics analysis and composed by 11,129 20"-PMT facing inwards with $40 \%$ photo-coverage

- The outer detector is used as veto for cosmic rays and made up of 1,884 8"-PMT facing outwards 


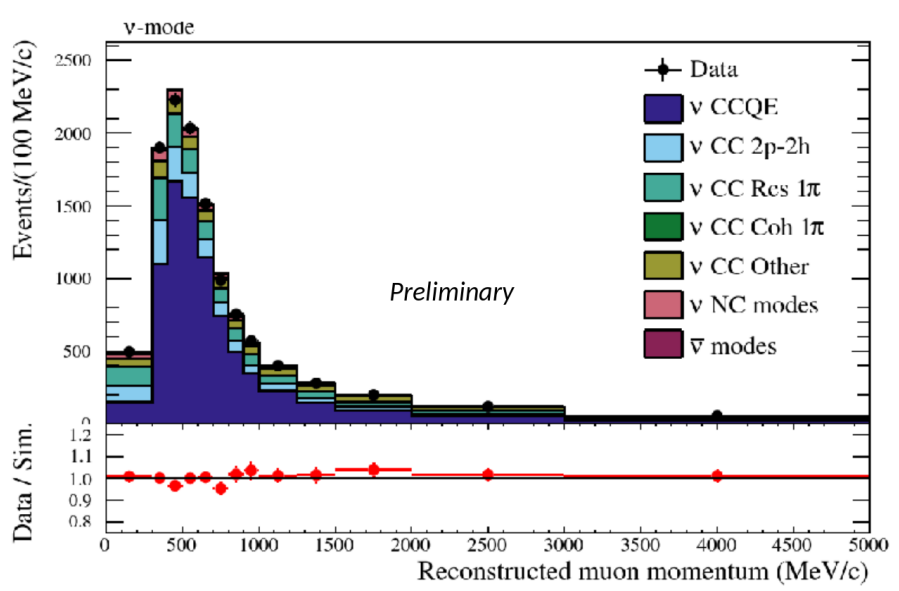

Figure 5: Comparison of data and simulated muon reconstructed spectrum at the T2K near detector, ND280.

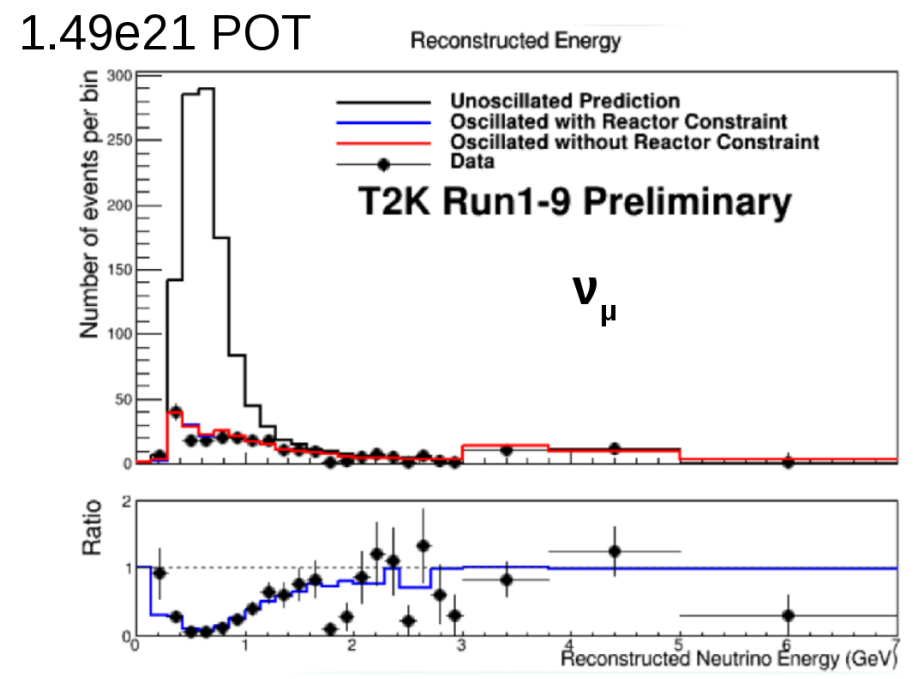

Figure 6: Comparison of reconstructed neutrino flux at the far detector of data and oscillated and unoscillated simulations.

Super-Kamiokande is currently being upgraded and refurbished for dissolving gadolinium (Gd) into the ultra-pure water (SuperK-Gd), enhancing hugely its neutron-tagging capabilities. SuperK-Gd will operate during T2K phase-II.

In addition, the NA61/SHINE hadron production experiment at CERN provides input for reducing the T2K flux uncertainties to $\sim 5 \%$.

\section{T2K neutrino oscillation results}

The analysis strategy for the oscillation parameters is to compare the observed event rates at SK with the predictions under oscillation hypothesis with inputs from the near detectors. The next table shows the rate prediction for different values of $\delta_{C P}$. 
Table 1: Best fit values of the T2K experiment oscillation analysis for $\sin ^{2} \theta_{23}$ and $\Delta m_{32}^{2}$ in both mass ordering scenarios.

\begin{tabular}{|c|c|c|}
\cline { 2 - 3 } \multicolumn{1}{c|}{} & Normal Ordering & Inverted Ordering \\
\hline $\sin ^{2} \theta_{23}$ & $0.536_{-0.046}^{+0.031}$ & $0.536_{-0.041}^{+0.031}$ \\
\hline$\Delta m_{32}^{2}\left(10^{-3} \mathrm{eV}^{2}\right)$ & $2.434 \pm 0.064$ & $2.410_{-0.063}^{+0.062}$ \\
\hline
\end{tabular}

\begin{tabular}{|c|c|c|c|c|c|}
\hline \multirow{2}{*}{ Sample } & \multicolumn{4}{|c|}{ Prediction } & \multirow{2}{*}{ Data } \\
\cline { 2 - 5 } & $\delta_{C P}=-\pi / 2$ & $\delta_{C P}=0$ & $\delta_{C P}=\pi / 2$ & $\delta_{C P}=\pi$ & \\
\hline FHC 1R(ing) $\mu$ & 268.5 & 268.2 & 268.5 & 268.9 & 243 \\
\hline RHC 1R(ing) $\mu$ & 95.5 & 95.3 & 95.5 & 95.8 & 102 \\
\hline FHC 1R $e$ 0 decay-e & 73.8 & 61.6 & 50.0 & 62.2 & 75 \\
\hline FHC 1R $e$ 1 decay-e & 6.9 & 6.0 & 4.9 & 5.8 & 15 \\
\hline RHC 1R $e$ 0 decay-e & 11.8 & 13.4 & 14.9 & 13.2 & 9 \\
\hline
\end{tabular}

The results for the $\theta_{23}$ and $\Delta m_{32}^{2}$ from T2K with reactor data constraints are shown in figure 7. These results show a preference for the $\theta_{23}$ second octant, although lower values are less than $2 \sigma$ away. Since the neutrino mass ordering is yet to be determined, the fit is done for both, normal and inverted, orderings.
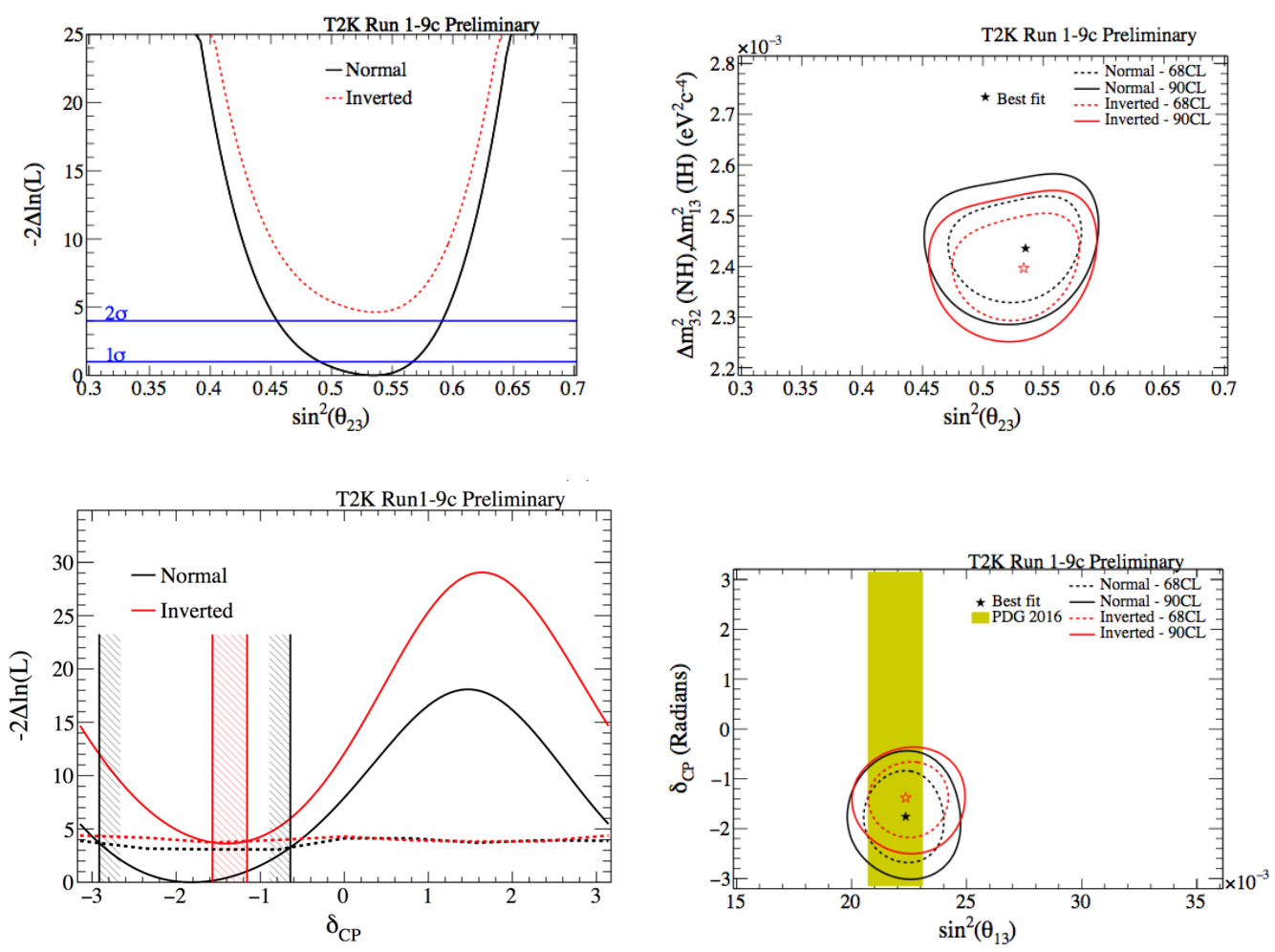

Figure 7: T2K latest results for the relevant oscillation parameters assuming the constraints given by reactor experiments and for both mass orderings. From left to right and from top to bottom: $\chi^{2}$ curve for $\theta_{23}, 2 \mathrm{D}$ contours for $\Delta m_{32}^{2}$ and $\theta_{23}, \chi^{2}$ curve for $\delta_{C P}$ and 2D contours for $\delta_{C P}$ and $\theta_{13}$.

The results for the $\delta_{C P}$ parameter show that the best fit is placed at large and negative values for both neutrino mass orderings, disfavouring CP-conserving values by more than the $2 \sigma$. 
Table 2: Best fit values of the T2K experiment oscillation analysis for the neutrino mass ordering and the CP-pahse.

\begin{tabular}{|c|c|c|}
\cline { 2 - 3 } \multicolumn{1}{c|}{} & Mass Ordering & $\delta_{C P}(\mathrm{rad})$ \\
\hline Best Fit & $\mathrm{NH}(2.0 \sigma)$ & $-0.57 \pi$ \\
\hline
\end{tabular}

\subsection{The NO $v$ A Experiment}

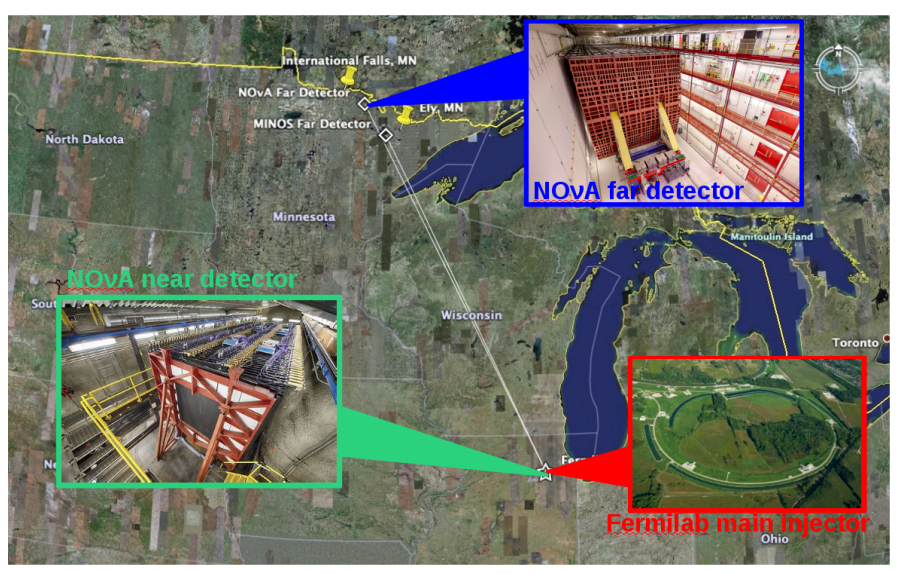

Figure 8: Geographical view of the NO $v$ A experiment layout.

The NO $v$ A experiment is located in the US. It is also composed by near and far detectors and uses the Fermilab main injector as neutrino source.

\section{The NO $v$ A beam}

The Fermilab neutrino main injector (NuMI) produces muon neutrinos and antineutrinos and can also run in neutrino or antineutrino mode. This is the most powerful neutrino beam in the world, it has been running at the nominal $700 \mathrm{~kW}$ power since January 2017. Since the start of the NOvA experiment, $8.85 \cdot 10^{20} \mathrm{POT}$ in neutrino mode and $6.91 \cdot 10^{20} \mathrm{POT}$ in antineutrino mode have been accumulated.

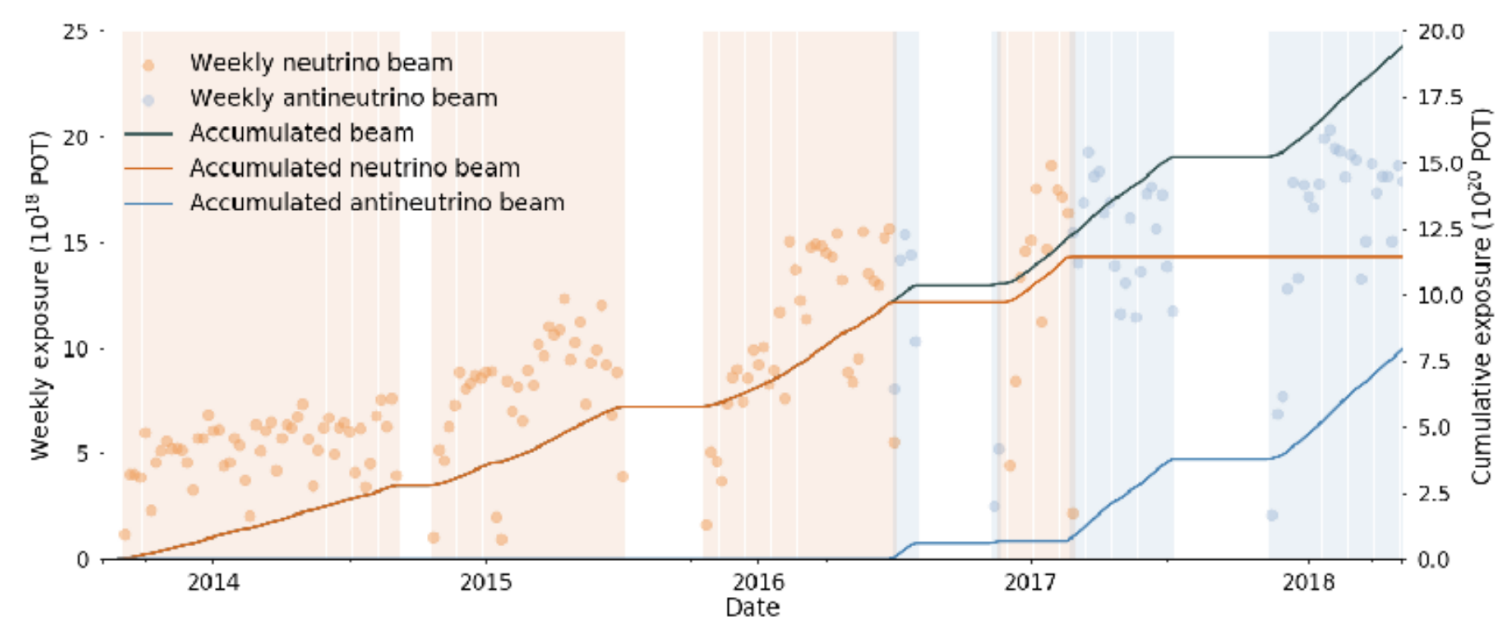

Figure 9: Accumulated POT of the NO $v$ A experiment with time in both, neutrino and antineutrino beam modes. 

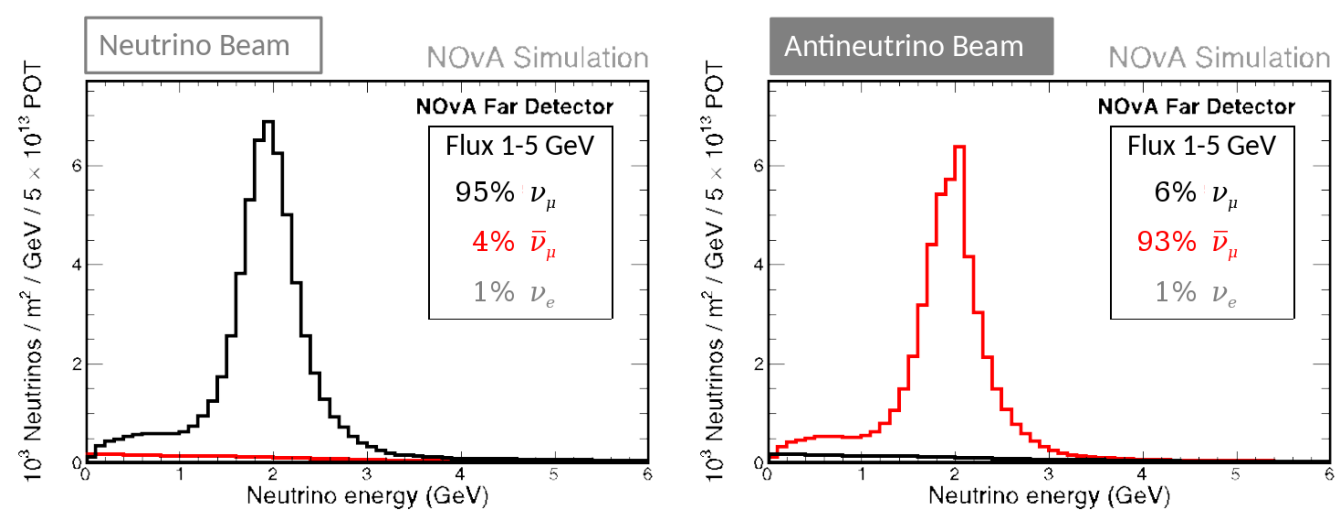

Figure 10: Neutrino spectrum simulation as seen by the NO $v$ A far detector assuming no oscillations.

The mean neutrino energy is $\left\langle E_{v}\right\rangle \sim 1.9 \mathrm{GeV}$ and directed $14.6 \mathrm{mrad}\left(\sim 0.84^{0}\right)$ off-axis with respect to the far and near detectors. As in $\mathrm{T} 2 \mathrm{~K}$, this technique provides a narrow neutrino spectrum at the appearance maximum and disappearance minimum, optimizing the measurement of the neutrino oscillation parameters.

\section{The NO $v$ A detectors}

The NO $v$ A experiment is composed by two identical, except for the size, detectors and located $1 \mathrm{~km}$ (near detector) and $810 \mathrm{~km}$ (far detector) from the neutrino production point. These detectors are tracking calorimeters made up of 344,000 cells of extruded and highly reflective plastic PVC filled with liquid scintillator. Each cell of the detectors is $3.9 \mathrm{~cm}$ wide, $6.0 \mathrm{~cm}$ deep.

- Near detector: Each cell is $3.9 \mathrm{~m}$ long and contains a total mass of 290 ton. To enhance muon containment, the downstream end has an additional ten layers of 10 -cm-thick steel plates.

- Far detector: Each cell is $15.5 \mathrm{~m}$ long and it contains 14 kton.

The signal from each liquid scintillator cell is read out through a wavelength-shifting fiber (WLS). The fiber-end readout consists of a single pixel of a 32 pixel avalanche photo diode (APD) array.

The near detector is located about $100 \mathrm{~m}$ underground, serving for reducing the systematic uncertainties related to the flux predictions and also conducts cross-section studies. The fact that near and far detectors share the same technology and setup is crucial for the understanding and cancellation of the systematic errors. 


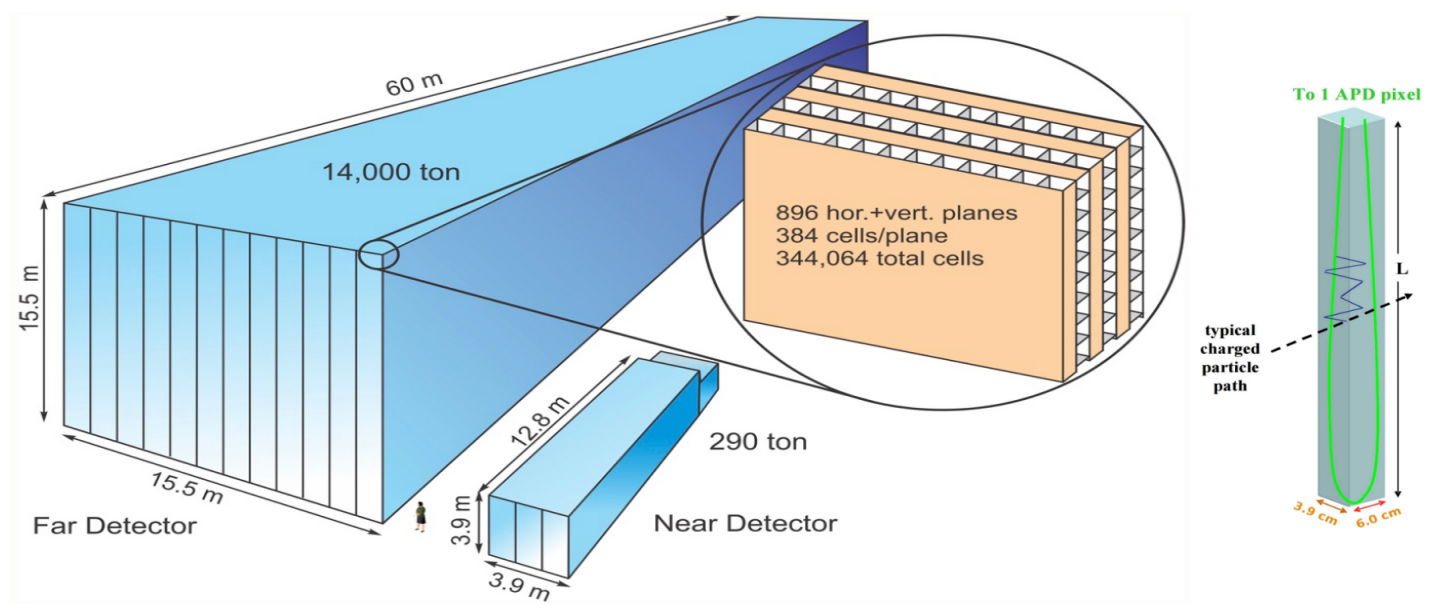

Figure 11: Schematic view of the NO $v$ A near and far detectors with their components.
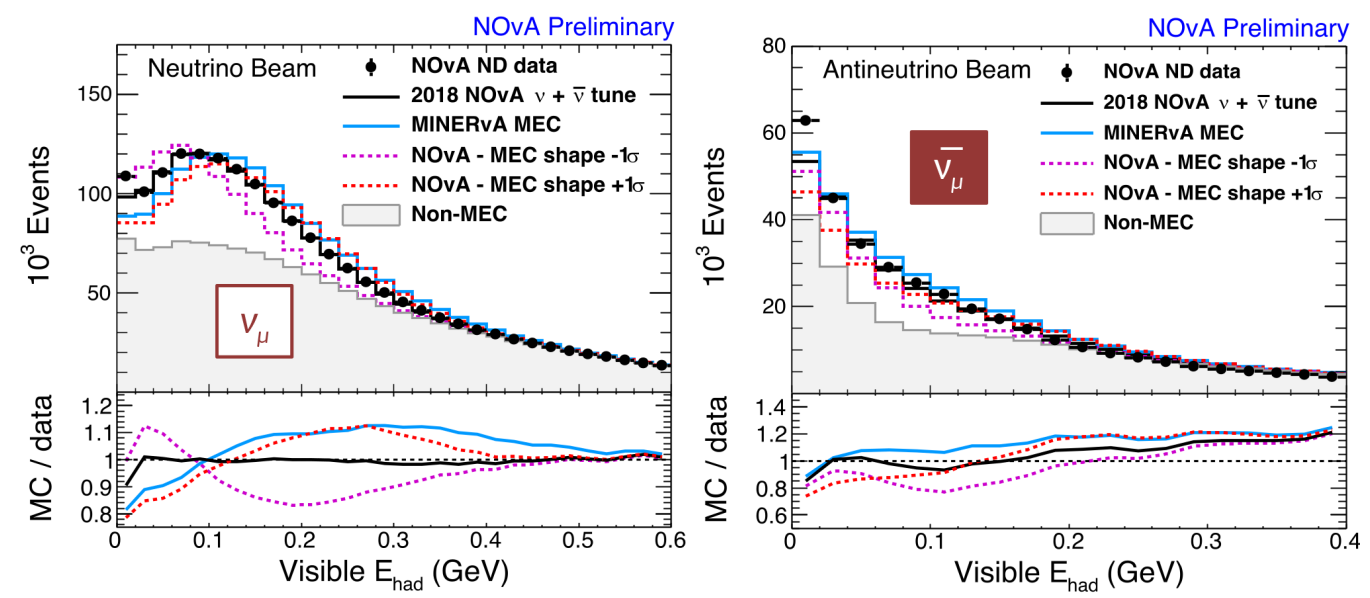

Figure 12: NO $v$ A neutrino spectrum at the near detector for both beam configurations. The Monte Carlo simulation is tuned according to the inputs from the data in order to obtain a more accurate simulation for the far detector neutrino flux and cross-section.

The far detector is placed on the surface under a concrete and barite overburden, which stops a significant fraction of the cosmic rays.

\section{NO $v$ A neutrino oscillation results}

Despite being very different experiments, the NO $v \mathrm{~A}$ and $\mathrm{T} 2 \mathrm{~K}$ analysis strategies are the same. The far detector event rates are compared with the oscillation predictions, and near detector data is used to reduce uncertainties related to the beam flux and the cross-section interactions.

Figure 14 shows different predictions for the NO $v$ A assuming different values of the neutrino oscillation parameters together with the actual data point in terms of the neutrino and antineutrino events rates. Data agrees with simulation showing a preference for the normal mass ordering and the second octant of $\theta_{23}$. 

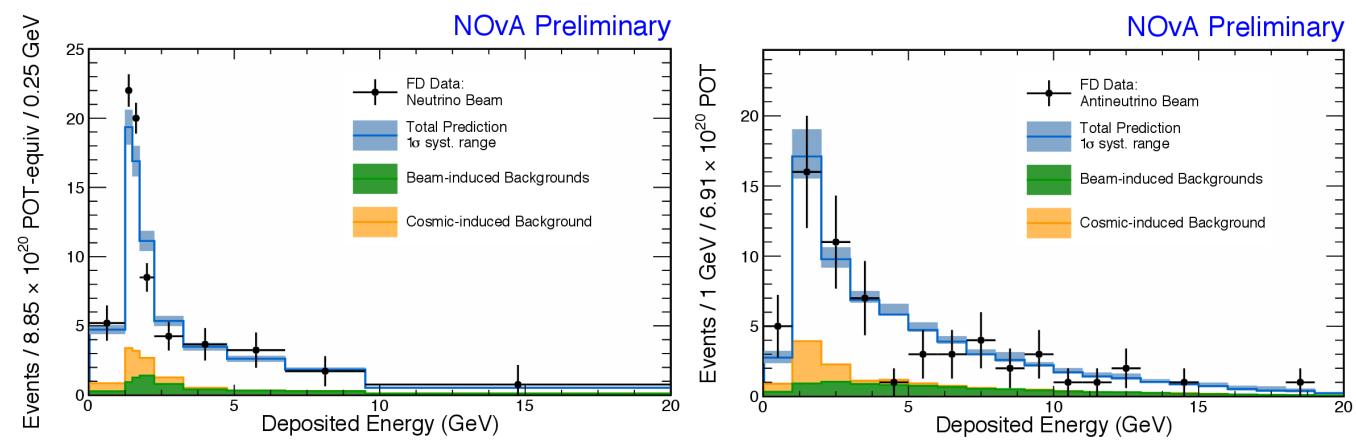

Figure 13: Simulation and data reconstructed neutrino spectrum at the far detector.
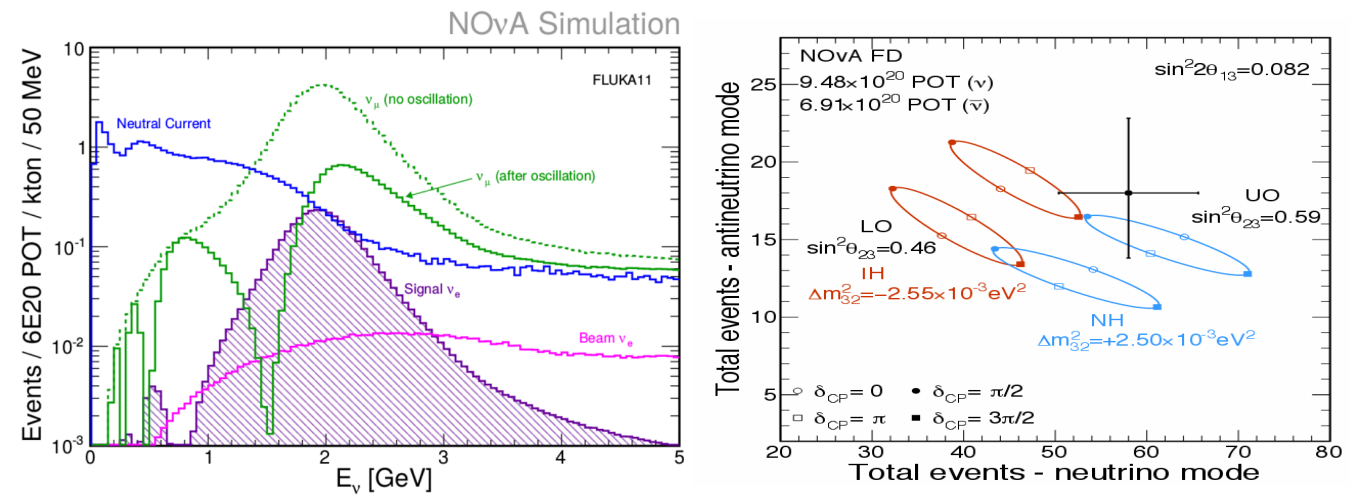

Figure 14: Left plot shows the reconstructed neutrino spectrum from simulation and data at the far detector. Right plot shows a comparison of oscillated event rates between data and Monte Carlo simulation.
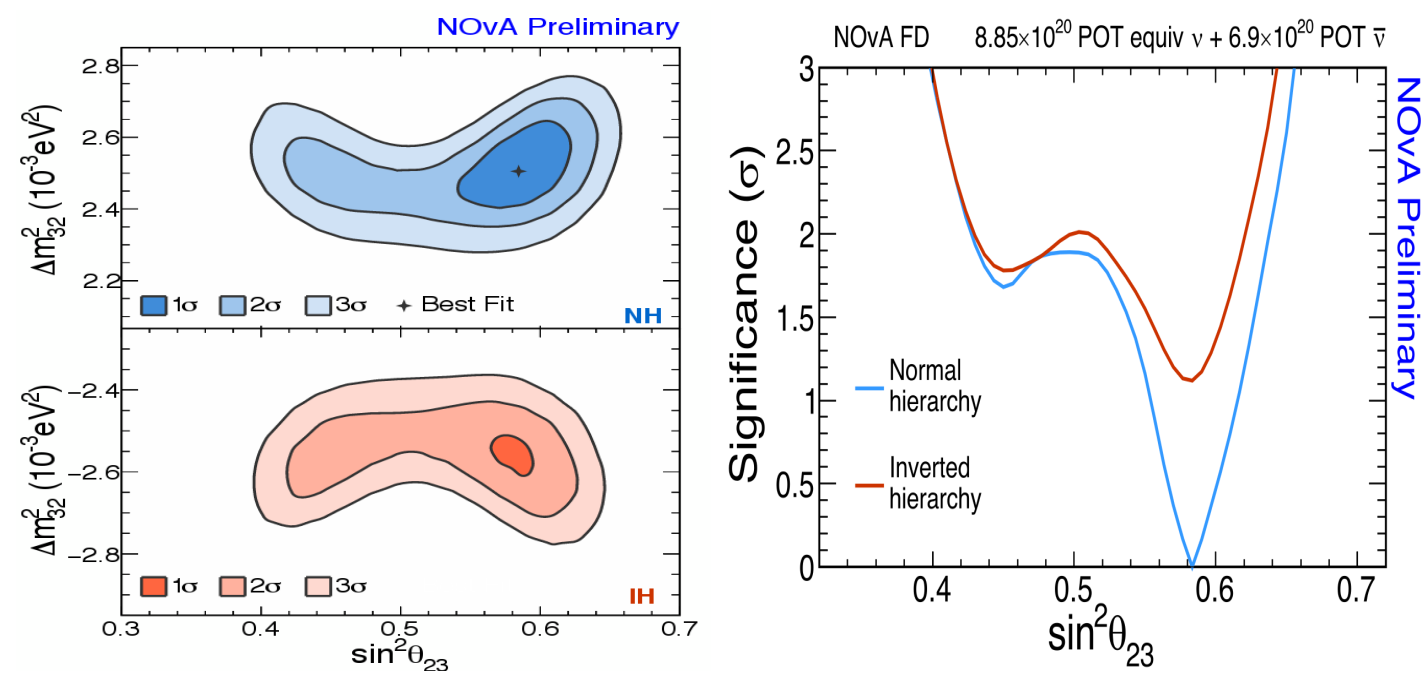

Figure 15: NO $v$ A latest results for $\Delta m_{32}^{2}$ and $\theta_{23}$. Left plot shows the 2D contours for the $\Delta m_{32}^{2}$ and $\theta_{23}$ parameters and assuming both neutrino mass orderings. Right plot shows the $\chi^{2}$ curve for $\theta_{23}$.

Next, the results of the NOvA experiment are summarized in figures 15 and 16. These results are in agreement with the previous ones from $\mathrm{T} 2 \mathrm{~K}$, this results in more complete scenario 

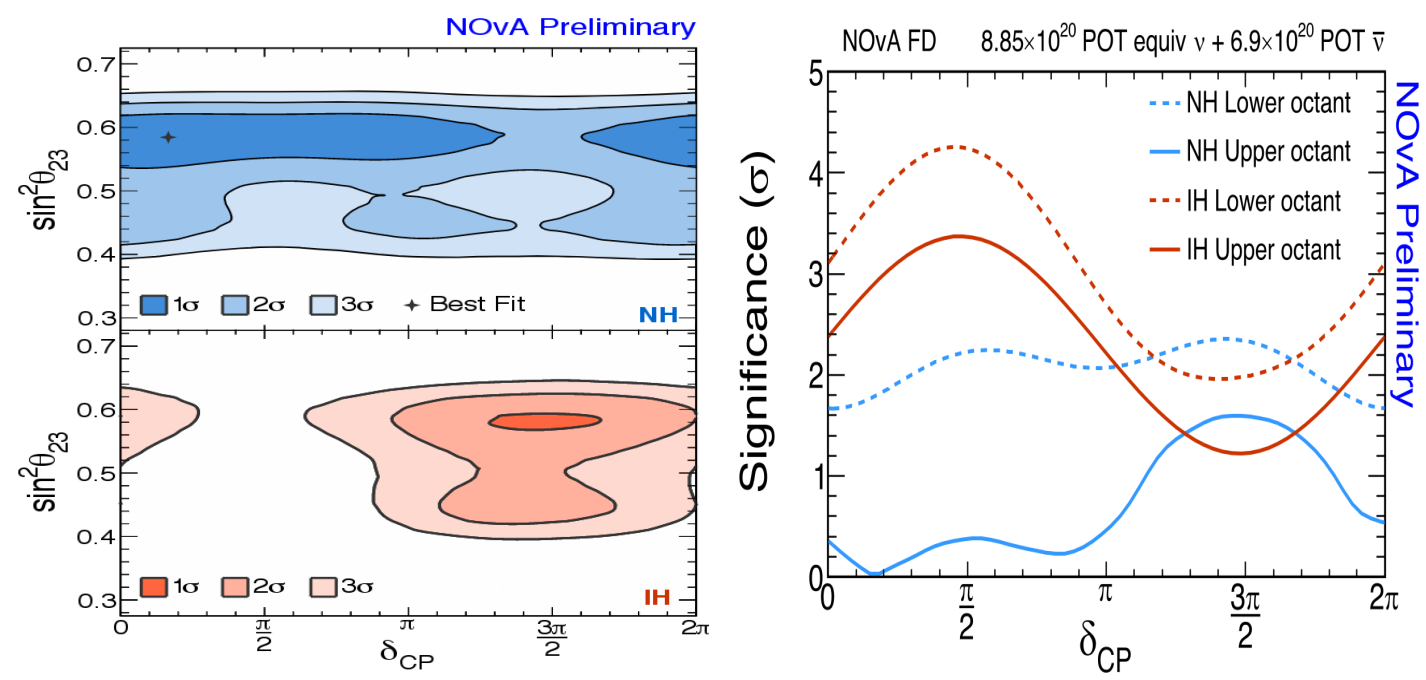

Figure 16: NO $v$ A latest results for $\theta_{23}$ and $\delta_{C P}$. Left plot shows the 2D contours for the $\theta_{23}$ and the $\delta_{C P}$ parameters and assuming both neutrino mass orderings. Right plot shows the $\chi^{2}$ curve for $\delta_{C P}$.

Table 3: Best fit values of the NOvA experiment oscillation analysis.

\begin{tabular}{|c|c|c|c|c|}
\cline { 2 - 5 } \multicolumn{1}{c|}{} & Mass Ordering & $\sin ^{2} \theta_{23}$ & $\Delta m_{32}^{2}\left(10^{-3} \mathrm{eV}^{2}\right)$ & $\delta_{C P}$ \\
\hline Best Fit & $\mathrm{NH}(1.8 \sigma)$ & $0.58 \pm 0.03$ & $2.51_{-0.08}^{+0.12}$ & $0.17 \pi$ \\
\hline
\end{tabular}

of the remaining unknowns of the neutrino oscillation parameters.

The normal mass ordering and the upper (second) octant of $\theta_{23}$ are preferred, and there is also a slight preference for values of $\delta_{C P}$ close to $\pi / 4$.

Table 3 shows the best fit values given by the current NO $v$ A data.

\subsection{T2K and NO $v$ A Joint Neutrino Oscillation Analysis}

Given the huge success of the T2K and NOvA experiments, it has been agreed, by the two collaborations, the formation of a working group to perform a combined analysis of their data in order to enhance the measurements of neutrino oscillation parameters made by each experiment individually. This joint analysis is foreseen for 2021 .

\subsection{The MINOS/MINOS+ Experiment}

MINOS was mainly dedicated to measure the atmospheric oscillation parameters and MINOS+ is more focused on the additional sterile neutrinos search.

\section{The MINOS and MINOS+ beam}

They use the NuMI beam, the same as the NOvA experiment:

- MINOS period: The neutrino mean energy is $\sim 3 \mathrm{GeV}$ and has accumulated during its operation $10.56 \cdot 10^{20}$ POT in $v$-mode and $3.36 \cdot 10^{20}$ POT in $\bar{v}$-mode.

- MINOS+ period: The neutrino mean energy is $\sim 7 \mathrm{GeV}$ and, so far, has accumulated $9.69 \cdot 10^{20} \mathrm{POT}$ in $v$-mode. 


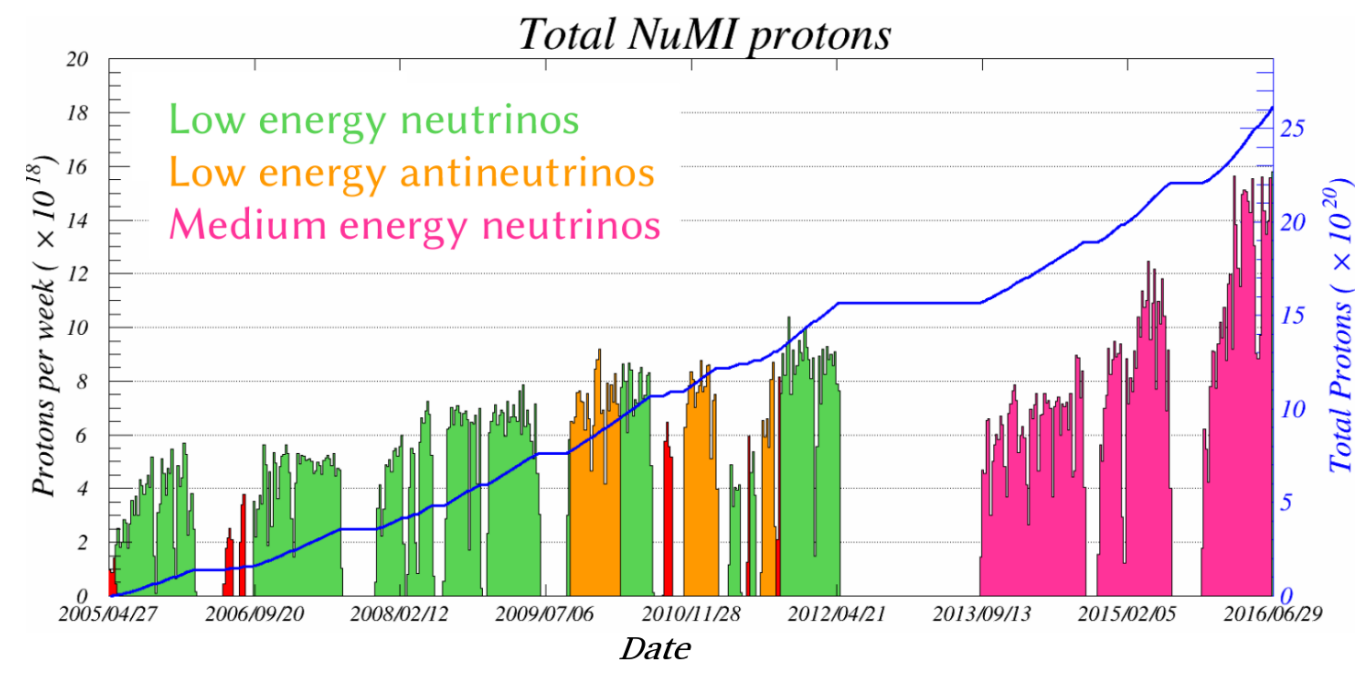

Figure 17: Accumulation of POT by the MINOS and MINOS+ experiments during their operation.

\section{The MINOS and MINOS+ detectors}

As happens for the NO $v$ A detector, both, near and far, detectors share the same technology, but in this case they are aligned with the beam axis, do not use the previously explained offaxis layout.

The two MINOS detectors are iron-scintillator tracking calorimeters which consist of planes of steel and planes of $1 \mathrm{~cm}$ thick plastic scintillator. Both detectors are magnetized with a $\sim 1.3 \mathrm{~T}$ magnetic field, allowing the identification of the charged particles and improving their energy reconstruction.
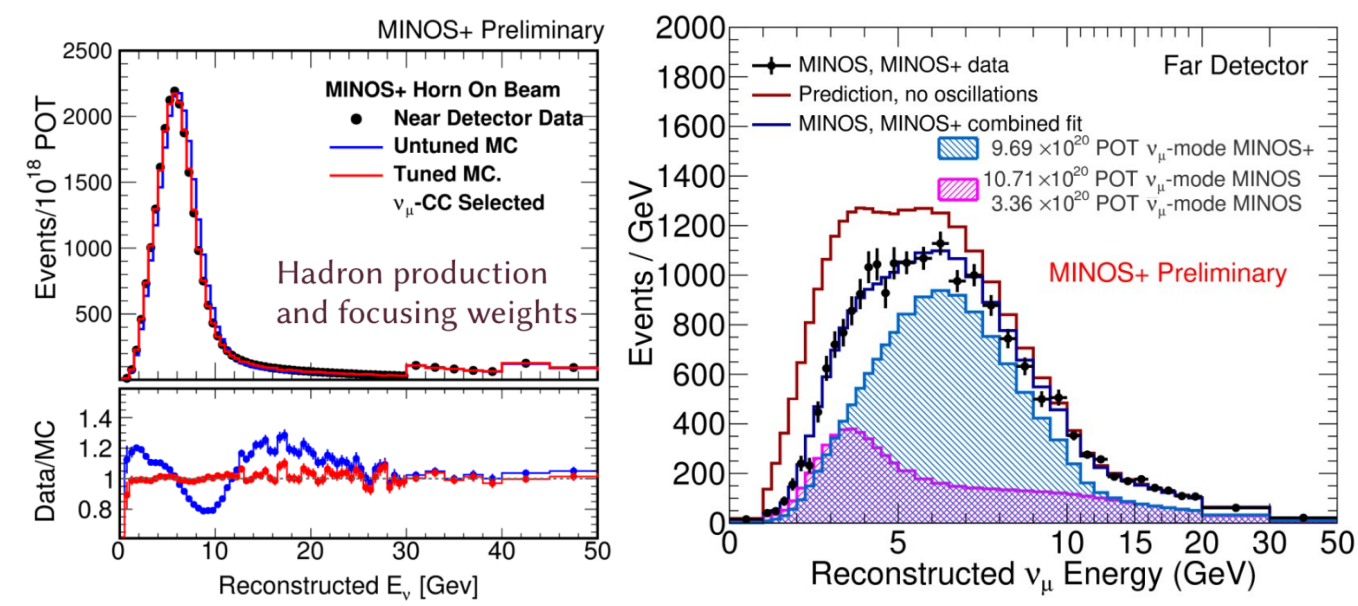

Figure 18: Comparison plots of the reconstructed neutrino spectrum at near and far detectors.

- Near Detector: It is located $1 \mathrm{~km}$ from the neutrino target and $100 \mathrm{~m}$ underground, containing 1 kton of mass.

- Far Detector: Located $735 \mathrm{~km}$ from the neutrino target in the Soudan mine, $716 \mathrm{~m}$ underground and has 5.4 kton mass. 
Table 4: Best fit values of the MINOS and MINOS+ experiments oscillation analysis.

\begin{tabular}{|c|c|c|c|}
\cline { 2 - 4 } \multicolumn{1}{c|}{} & Mass Ordering & $\sin ^{2} \theta_{23}$ & $\Delta m_{32}^{2}\left(10^{-3} \mathrm{eV}^{2}\right)$ \\
\hline Best Fit & NH (slight pref.) & 0.42 & 2.42 \\
\hline
\end{tabular}

As in the previous experiments, the near detector has a crucial role in reducing the systematic uncertainties at the far detector, particularly in this case, where both detectors share the same technology.

\section{MINOS and MINOS+ neutrino oscillation results}

The analysis strategy of MINOS and MINOS+ for the measurement of neutrino oscillation parameters is similar to $\mathrm{T} 2 \mathrm{~K}$ and $\mathrm{NO} v \mathrm{~A}$ experiments.

Figure 19 shows the current results from the combined analysis of MINOS and MINOS+.
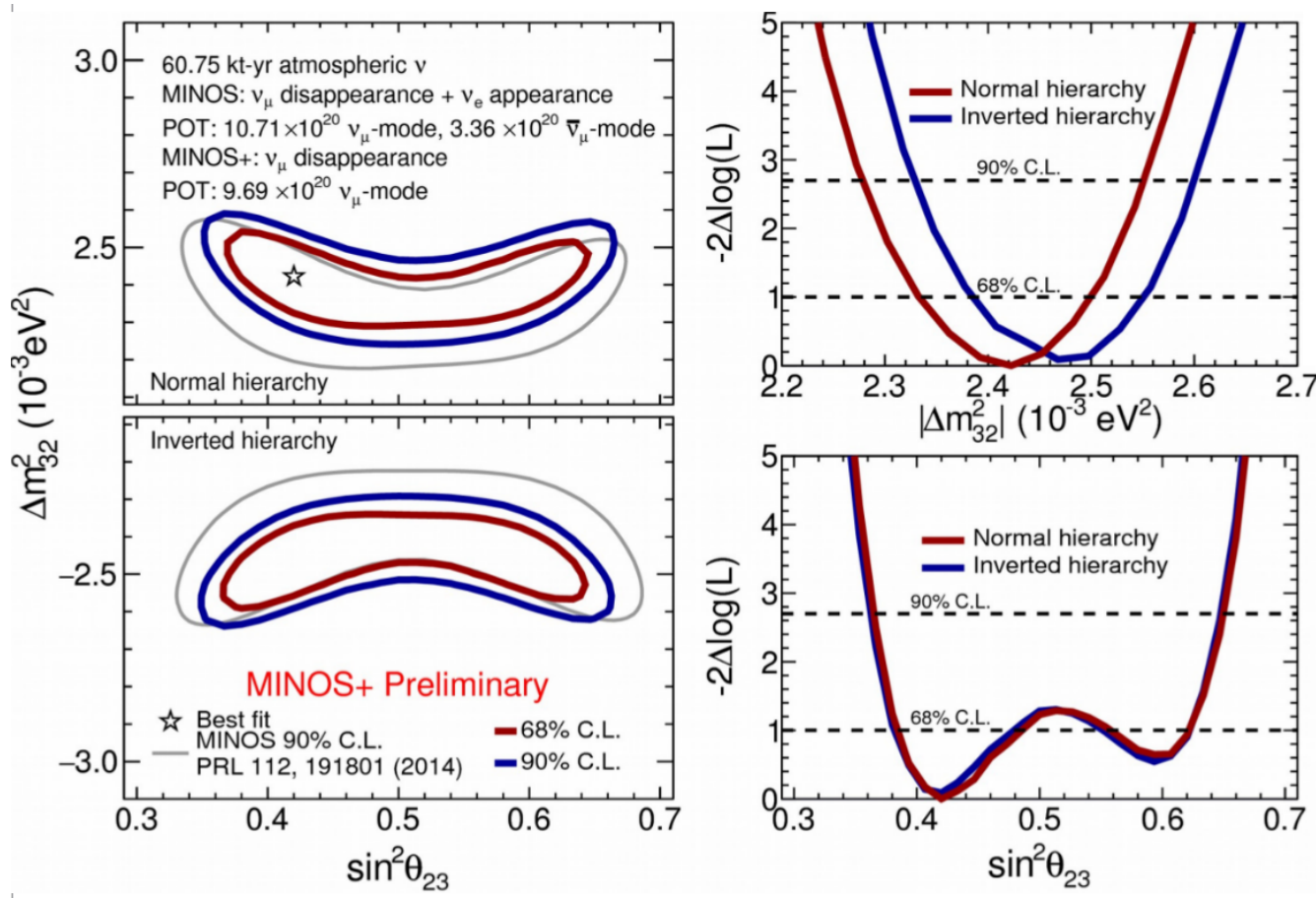

Figure 19: MINOS and MINOS+ latest combined results for $\theta_{23}$ and $\Delta m_{32}^{2}$. Left plot shows the 2D contours and best fit point for the $\Delta m_{32}^{2}$ and the $\theta_{23}$ assuming both neutrino mass orderings. Right plots show the $\chi^{2}$ curves for the $\Delta m_{32}^{2}$ (top) and $\theta_{23}$ (bottom).

The value of $\Delta m_{32}^{2}$ is consistent with the best fit values of the previous experiments and the results show a preference for the first octant of the $\theta_{23}$, but values in the second octant are less than the $1 \sigma$ away from the best fit point.

Finally, results also show a very slight preference for the normal neutrino mass ordering. 


\section{Results from Current LBL Neutrino Oscillation Experiments}

Figure 20 summarizes the the results from all the reviewed experiments for the $\Delta m_{32}^{2}$ and $\theta_{23}$ oscillation parameters.

All the described experiments show a preference for the normal ordering of neutrino masses for which the data is becoming more compelling. As for the CP phase, T2K shows a preference for values close to $3 \pi / 2(-\pi / 2)$, whereas the NO $v$ A experiment prefer smaller values, both disfavouring CP-conserving values.

These results show the need for the T2K and NO $v \mathrm{~A}$ joint analysis because it will provide a more clear picture of the remaining neutrino oscillation parameters.

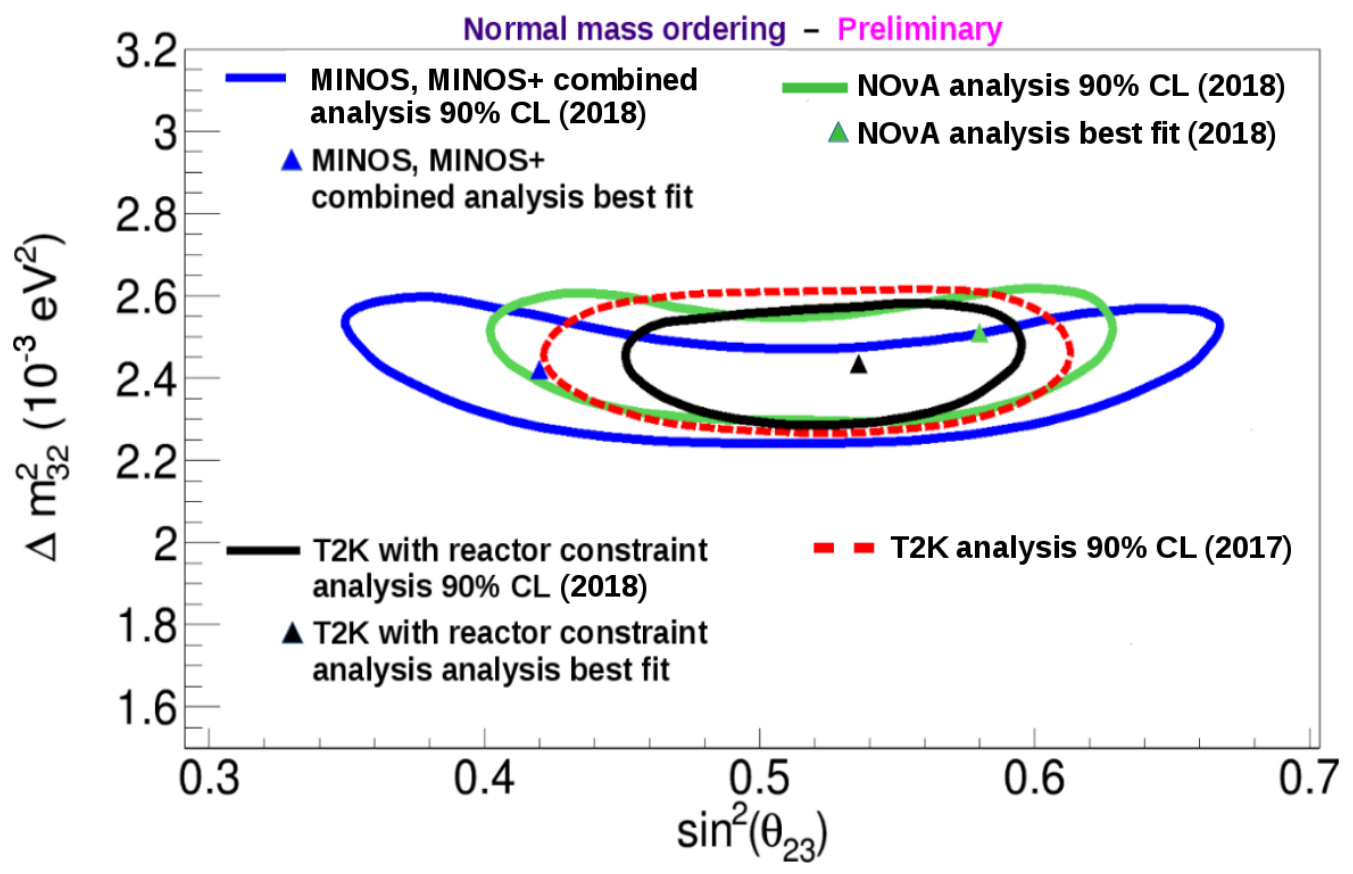

Figure 20: $90 \%$ confidence level 2D contours and best fit points for the $\Delta m_{32}^{2}$ and $\theta_{23}$ for the T2K (dashed red), the T2K with reactor constraints (black), the NO $v \mathrm{~A}$ experiment and the MINOS and MINOS+ experiments.

\section{Conclusions}

The three main, currently operating, LBL neutrino experiments, T2K, NO $v$ A and MINOS/MINOS+ have been reviewed and their most important results for the three neutrino oscillations shown. In terms of neutrino oscillation results, all three experiments show a preference for normal neutrino mass ordering and $\mathrm{NO} v \mathrm{~A}$ and T2K data prefer the second octant of $\theta_{23}$ and disfavour CP-conserving values of $\delta_{C P}$. MINOS and MINOS+ best fit is at the first octant of $\theta_{23}$, although compatible with the second octant preference from the other experiments at $<1 \sigma$.

T2K will keep taking data until 2026 with improved neutrino beam and near and far detectors with the goal of $20 \cdot 10^{21} \mathrm{POT}$ accumulated. In addition, a proposed joint fit of NO $v \mathrm{~A}$ and T2K data will improve the sensitivity for the neutrino oscillation parameters, with special emphasis for the CP phase.

All these experiments are currently running and will continue to acquire data until the next generation experiments become operational, setting the basis to achieve precise measurements of all the neutrino oscillation parameters. 


\section{Acknowledgments}

I would like to thank the organizers of the $15^{\text {th }}$ Tau Lepton Physics Workshop the T2K collaboration and the NO $v \mathrm{~A}$ and MINOS experiments for giving me the opportunity to give this review talk dealing with the current status of the neutrino oscillation research within long-baseline experiments.

Funding information This has been partially funded by the project Física de oscilaciones de neutrinos en el IFIC y la UAM, FPA2016-78417-C2-1-P, of the Spanish state agency, Ministerio de Economia y Competitividad.

\section{References}

[1] B. Pontecorvo, Mesonium and antimesonium, J. Exptl. Theoret. Phys. (U.S.S.R) 33, 549 (1957).

[2] S. Mikheyev et al., Resonance enhancement of oscillations in matter and solar neutrino spectroscopy, Yadernaya Fizika 42, 1441 (1985).

[3] K. Abe et al. [T2K Collaboration], Search for CP violation in neutrino and antineutrino oscillations by the T2K experiment with $2.2 \times 1021$ protons on target, Phys. Rev. Lett. 121, 171802 (2018), doi:10.1103/PhysRevLett.121.171802.

[4] K. Abe et al. [T2K Collaboration], The T2K experiment, Nucl. Instrum. Methods Phys. Res. Sect. A 659, 106 (2011), doi:10.1016/j.nima.2011.06.067.

[5] [T2K Collaboration], http://t2k-experiment.org/2018/01/t2k-nova-announce

[6] K. Abe et al. [T2K Collaboration], Measurement of the $v_{\mu}$ charged-current quasielastic cross section on carbon with the ND280 detector at T2K, Phys. Rev. D 92, 112003 (2015), doi:10.1103/PhysRevD.92.112003.

[7] K. Abe et al. [T2K Collaboration], Measurement of $\bar{v}_{\mu}$ and $v_{\mu}$ charged current inclusive cross sections and their ratio with the T2K off-axis near detector, Phys. Rev. D 96, 052001 (2017), doi:10.1103/PhysRevD.96.052001.

[8] M. A. Acero et al. [NO $v$ A Collaboration], New constraints on oscillation parameters from $v_{e}$ appearance and $v_{\mu}$ disappearance in the NOvA experiment, Phys. Rev. D 98, 032012 (2018), doi:10.1103/PhysRevD.98.032012.

[9] M. A. Acero et al. [NO $v$ A Collaboration], New constraints on oscillation parameters from $v_{e}$ appearance and $v_{\mu}$ disappearance in the NOvA experiment, Phys. Rev. D 98, 032012 (2018), doi:10.1103/PhysRevD.98.032012.

[10] P. Adamson et al. [NO vA Collaboration], Measurement of the neutrino mixing angle $\theta_{23}$ in NOvA, Phys. Rev. Lett. 118, 151802 (2017), doi:10.1103/PhysRevLett.118.151802.

[11] P. Adamson et al. [NOvA Collaboration], First measurement of electron neutrino appearance in NOvA, Phys. Rev. Lett. 116, 151806 (2016), doi:10.1103/PhysRevLett.116.151806.

[12] P. Adamson et al. [MINOS Collaboration], The NuMI neutrino beam, Nucl. Instrum. Methods Phys. Res. Sect. A 806, 279 (2016), doi:10.1016/j.nima.2015.08.063. 
[13] P. Adamson et al. [MINOS Collaboration], Combined analysis of $v_{\mu}$ disappearance and $v_{\mu} \rightarrow v_{e}$ appearance in MINOS using accelerator and atmospheric neutrinos, Phys. Rev. Lett. 112, 191801 (2014), doi:10.1103/PhysRevLett.112.191801.

[14] P. Adamson et al. [MINOS Collaboration], Electron neutrino and antineutrino appearance in the full MINOS data sample, Phys. Rev. Lett. 110, 171801 (2013), doi:10.1103/PhysRevLett.110.171801.

[15] D. G. Michael et al. [MINOS Collaboration], The magnetized steel and scintillator calorimeters of the MINOS experiment, Nucl. Instrum. Methods Phys. Res. Sect. A 596, 190 (2008), doi:10.1016/j.nima.2008.08.003. 